\title{
Study of peculiarities of the thermal expansion of zirconium thin films by molecular-dynamics simulation
}

\author{
E.B. Dolgusheva and V.Yu. Trubitsin \\ Physical-Technical Institute, Ural Branch of Russian Academy of Sciences, 132 Kirov Str.,426001 Izhevsk, Russia
}

\section{Abstract}

The peculiarities of thermal expansion of $b c c$ and $f c c$ zirconium films with (100) and (110) crystallographic orientations are studied at a constant zero pressure by the molecular dynamics (MD) method with a many-body interatomic interaction potential obtained in the embedded atom model. It is shown that after relaxation the cubic lattices become tetragonal (bct and $f c t$ ), and for the metastable $f c t$ films the linear coefficients of thermal expansion in the film plane have a negative value in a wide temperatuThe peculiarities of thermal expansion of $b c c$ and $f c c$ zirconium films with (100) and (110) crystallographic orientations are studied at a constant zero pressure by the molecular dynamics (MD) method with a many-body interatomic interaction potential obtained in the embedded atom model. It is shown that 'after relaxation the cubic lattices become tetragonal (bct and $f c t$ ), and for the metastable $f c t$ films the linear coefficients of thermal expansion in the film plane have a negative value in a wide temperature range. The total and local vibrational 'density of states (VDOS) polarized along the $x, y, z$ axes is calculated for the surface and interior layers of $b c t$ and $f c t$ Zr films as a function of the temperature. It is shown that the peculiarities of the behavior of the vibrational density of states of surface atomic layers manifest themselves in the anisotropy of the changes of the film lattice parameters with temperature variation. A decrease in the lattice parameters with increasing temperature is observed in the directions where there occurs a softening of the local vibrational modes. re range. The total and local vibrational density of states (VDOS) polarized along the $x, y, z$ axes is calculated for the surface and interior layers of bct and $f c t$ Zr films as a function of the temperature. It is shown that the peculiarities of the behavior of the vibrational density of states of surface atomic layers manifest themselves in the anisotropy of the changes of the film lattice parameters with temperature variation. A decrease in the lattice parameters with increasing temperature is observed in the directions where there occurs a softening of the local vibrational modes.

Keywords:Thin films, Thermal-expansion, MD simulation

\section{Introduction}

In recent years, an ever increasing number of papers have been devoted to the study of so-called "negative" materials having negative coefficients of thermal expansion [1, 2, 3, 4]. In 1996 the research team led by John Evans published the paper [1] in which a negative co'efficient of thermal expansion (CTE) was found for the $\mathrm{Zr} \mathrm{W}_{2} \mathrm{O}_{8}$ ceramics in a wide range of temperatures from $0.3 K$ to $1050 K$. Later many other compounds with similar properties, named Negative Thermal Expansion (NTE) materials, have been discovered [5].

These materials with unusual properties are interesting not only theoretically, but also from an industrial standpoint. Materials with controlled thermal expansion can find several practical applications, such as switches and sensors, while materials with zero thermal expansion in a wide temperature range will be used in high-precision engineering. In instrument-making industry materials with

Email address: elena@ftiudm.ru (E.B. Dolgusheva and V.Yu. Trubitsin) strictly regulated values of linear CTE in specified temperature ranges of operation are in great demand. Recently the Evans team has created a material whose properties can be controlled throughout the thermal expansion range: from positive through zero to negative CTE [6]. The composite oxide $\mathrm{ZrMo}_{2} \mathrm{O}_{8}$ is a well studied compound distinguished by a negative $\mathrm{CTE}$, whereas the tincontaining analog of this cubic material, $\mathrm{SnMo}_{2} \mathrm{O}_{8}$, expands when heated. The authors of that paper state also to have demonstrated the possibility of introducing various amounts of zirconium into the lattice so that the obtained ceramic material can have a positive, negative or zero CTE. In essence the "positive" nature of the tincontaining material is balanced by "negative" zirconium, both metals being key elements of the same crystalline structure. Additionally, in Refs. $[7,8]$ it was shown by inelastic neutron scattering that in NTE structures a softening is observed in the VDOS curve. Thus a negative thermal expansion coefficient is observed in materials which are in a metastable state (for example, near a structural transition), have a strongly anharmonic character of in- 
teratomic interaction, and/or a softening of the phonon modes is evidenced in the VDOS curve, which results in a negative value of the Grüneisen parameter.

Zirconium is known to be a strongly anharmonic metal. As we see, it plays a decisive role in the formation of negative CTE in the NTE structures. We believe, then, that the study of the thermal expansion of $\mathrm{Zr}$ nanofilms is an important task, because elucidation of the dependence of linear CTE on the temperature and film thickness will make it possible to reveal the peculiarities of the formation of thermodynamical properties of nanofilms and draw some conclusions about the influence of the surface structure on their properties. Clarification of the nature of special physical features of nanosystems is of interest for both fundamental problems of the condensed matter physics and possible practical applications.

Earlier [9], we constructed a phase diagram Temperature-Thickness for zirconium films. This diagram presents the regions of final structures stable for no less than $1 \mathrm{~ns}$, one time step being equal to 1 fs. Here we should mention some important points. Firstly, the size effect was detected. Two characteristic sizes may be noted. The first one is of 5 unit cells $(\sim 2 \mathrm{~nm})$ : up to this size the film just falls apart, and the second critical size is the film thickness of 17 unit cells $(\sim 6.1 \mathrm{~nm})$ at which the mechanism and sequence of structural transformations change: from this size on the bcc (001) film remains stable in a sertain temperature range. Secondly, what is especially important, structures not observed in pure $\mathrm{Zr}$ in the usual bulk state were found. These are $f c c$ and orthorhombic structures. Recall that at atmospheric pressure only two phases are observed in Zr bulk samples: a high-temperature $b c c$ phase, and an $h c p$ phase found below $1136 K$. Thus, zirconium provides a possibility of studying the properties of a strongly anharmonic material existing in both stable and metastable states, in conditions close to structural transformations.

The molecular dynamics method makes it possible to determine the linear CTE in different crystallographic directions, namely, in the direction normal to the film surface, and in the plane of the film atomic layers, which allows the study of the film thermal expansion anisotropy. Furthermore, this method enables the investigation of physical characteristics, such as the lattice dynamics, separately for the surface and interior atomic layers of the film. In this work the MD method is used to study the peculiarities of thermal expansion of the zirconium thin films with cubic $(b c c, f c c)$ crystal structures for different crystallographic orientations of the surface at a constant zero pressure. We also performed a comparison of the temperature behavior of the lattice parameters and vibrational density of states of surface and interior atomic layers, polarized both in the film plane and in the direction normal to the film surface.
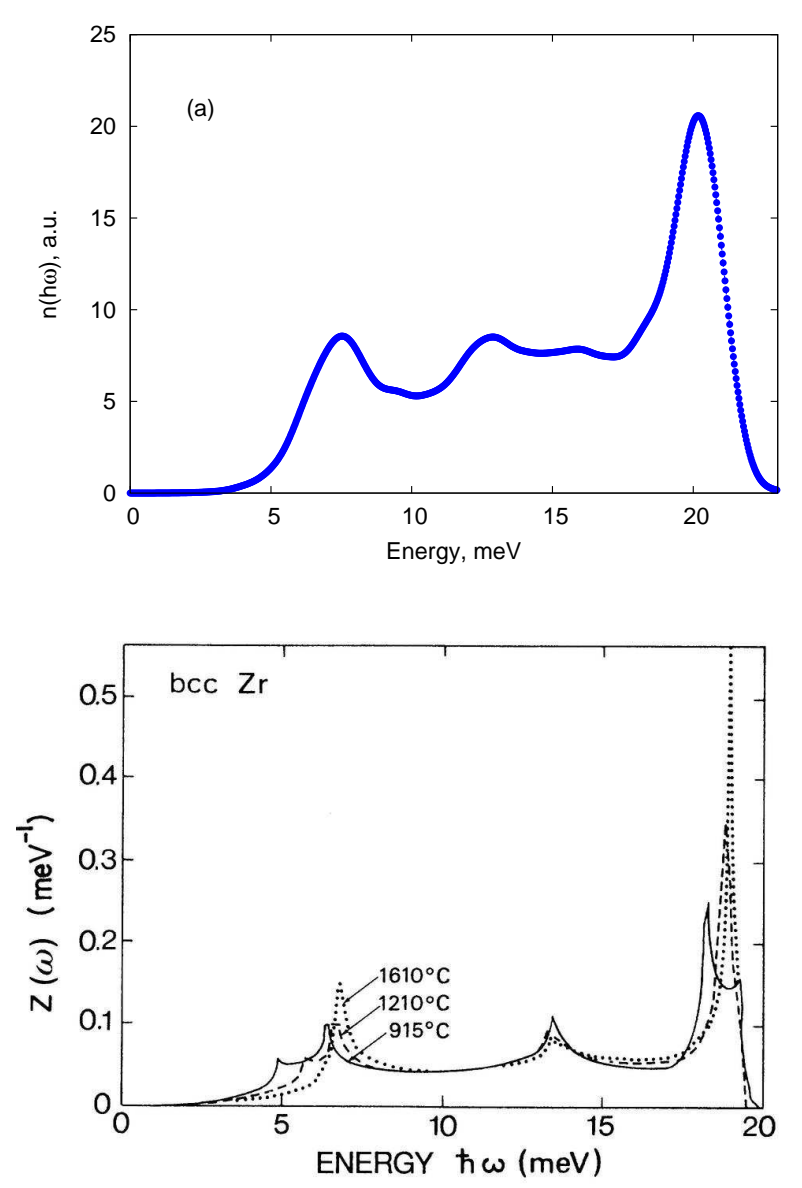

Figure 1: Vibrational density of states of bcc Zr. (a) calculated for a crystallite with cyclic boundary conditions at $\mathrm{T}=900 \mathrm{~K}$; (b) experimental values [14].

\section{Calculation method}

The physical characteristics of zirconium films were studied by the MD method using the standard XMD package [10]. The many-body potential (\#2) from Ref.[11] constructed within the embedded atom model [12] was chosen to describe the interatomic interaction in zirconium. In Ref.[11] it was shown that this potential allows one to obtain, to a high degree of accuracy, the bcc and hcp lattice parameters of zirconium, cohesive energy, elastic constants, melting temperature, and other physical characteristics. The bulk phonon dispersion curves calculated with this potential along the symmetrical directions of the Brillouin zone of $b c c$ zirconium at different temperatures were obtained in our paper [13]. A comparison with the experimental data shows that the potential chosen allows one to reproduce the experimentally observed features of the $\mathrm{Zr}$ phonon spectrum including the softening of the transverse N-phonon with decreasing temperature, and so it may be successfully used in calculating vibrational and thermal characteristics of zirconium films.

In this paper we present only the vibrational density of states for bulk zirconium (see Fig.1) as compared with the 
experiment [14]. The total VDOS, $g(\omega)$, was calculated as the Fourier transform of the autocorrelation velocity function, the velocity was averaged over all atoms on an interval of 10 ps after a relaxation period of 100 ps at a given temperature:

$$
g(w)=\int d t \frac{\sum_{i=1}^{N}\left\langle\mathbf{v}_{\mathbf{i}}(t) \mid \mathbf{v}_{\mathbf{i}}(0)\right\rangle}{\sum_{i=1}^{N}\left\langle\mathbf{v}_{\mathbf{i}}(0) \mid \mathbf{v}_{\mathbf{i}}(0)\right\rangle} \exp (i w t)
$$

Here $\mathbf{v}_{\mathbf{i}}(t)$ and $\mathbf{v}_{\mathbf{i}}(0)$ are the velocities of the $i$-th atom at time $t$ and the initial time, respectively. $<>$ denotes the averaging over time and the sum is taken over all atoms. For the calculation of the local VDOS (for example, the surface atoms VDOS) in the sum were considered only the velocities of the surface layer atoms. The local VDOS then were multiplied by the weight coefficient. To obtain the polarized VDOS all the velocities were multiplied by the polarization vector $\mathbf{p}$ in calculating the autocorrelation function:

$$
\mathbf{v}_{\mathbf{p}}(t)=\sum_{i=1}^{N} \mathbf{p} \cdot \mathbf{v}_{\mathbf{i}}(t)
$$

As seen from Fig.1, the positions of the main peaks are in good agreement with the experiment.

In calculating the lattice parameters, the coordinates of all the crystallite atoms were first averaged over a time interval of $50 \mathrm{ps}$, then the average values were taken over the atomic layer, thereafter the distance between the atomic layers in the given direction $(x, y, z)$ was found.

Note that in calculating the interlayer distance along the $z$ axis the near-surface layers ( 5 layers below the surface on each side) were excluded from consideration because of the fact that after relaxation near the film surface significant deviations from the interlayer distance values in the film interior are observed.

The coefficients of thermal expansion were calculated as follows:

$$
\beta=\frac{1}{V} \frac{d V}{d T}
$$

where $\beta$ is the film CTE; $V$ is the average volume per atom only in the film interior at the initial temperature; $d V$ is the relative change of this volume on heating the crystallite by $d T$ degrees. For the calculation of the linear CTE from our simulation we used the following expression:

$$
\alpha_{x, y, z}=\frac{1}{L_{X, Y, Z}} \frac{d L_{X, Y, Z}}{d T} .
$$

Here $\alpha_{z}$ is the linear CTE in the direction normal to the film plane along the $z$ axis; $\alpha_{x, y}$ are the linear CTE in the film plane along the $x$ and $y$ axes; $L$ is the average distance between the atomic layers at the initial temperature along the direction chosen; $d L$ is the relative change of this distance on heating the crystallite by $d T$ degrees.

\section{Lattice Parameters and Vibrational Density of States}

Note at once that the lattice cubic symmetry in $b c c$ and $f c c$ films changes after relaxation to tetragonal. As a result, the $b c c$ films turn into bct, and $f c c$ into $f c t$ ones.

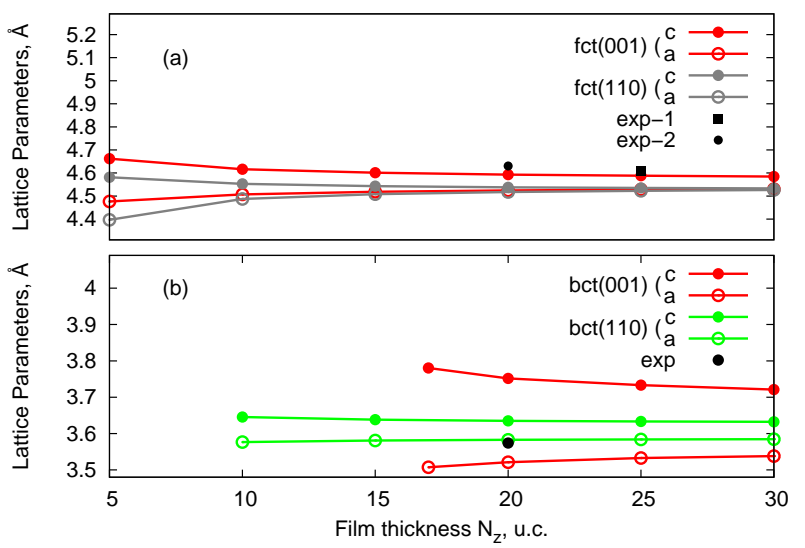

Figure 2: Lattice parameters $a$ and $c$ for the films after relaxation: (a) for $f c c$ films: (b) for $b c c$ films. The experimental values for $f c c$ Zr film were obtained: in [15, 16] (exp-1); in [17] (exp-2), and in [18] for a bulk bcc Zr sample.

Figure 2 presents the lattice parameters $a$ and $c$ averaged over $50 \mathrm{ps}$ for films of different thickness indicated on the abscissa in unit cells (u.c.) of the corresponding structures. For $f c t$ films the calculation was performed at $T=300 K$ (Fig.2(a)), and for bct films at $T=900 K$ (Fig.2(b)). The statistical error was $0.001 \AA$ at $300 K$ and $0.002 \AA$ at $900 K$. The lattice parameters for $f$ ct films with (001) and (110) crystallographic orientations are rather close in magnitude, while for bct (001) and (110) films they differ significantly. The bct (001) film is stabilized only from a thickness of 17 unit cells $\left(N_{z}=17 u\right.$.c. $)$. In the same figure are shown the experimental lattice parameters obtained in Refs. [15, 16] (4.61 $\AA$ indicated by black

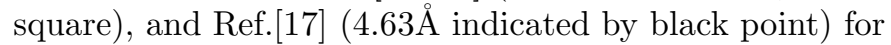

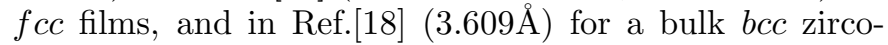
nium structure (fig.2(b)). The tetragonal distortion parameter, $c / a$, decreases with increasing film thickness. For $f c t$ films it varies from 1.042 to 1.011 . The tetragonality of $f$ ct zirconium films was also observed experimentally [16] with a tetragonal distortion parameter $c / a$ equal to $1.0220 \pm 0.022$.

The thickness has a determining effect on various properties of the films, which is due to their structural peculiarities and the arising mechanical stresses. We calculated the averaged distances between atomic layers in films of different thickness along the direction normal to the film plane, i.e. from one free surface to the other. The size of the basic crystallite along the $x$ and $y$ axes in all cases was equal to 24 u.c. The bct films were considered at $T=900 K$, and the $f c t$ ones at $T=300 K$. 
As shown by the calculations, significant variations in the average distance between atomic layers are observed in the interface regions of all films after relaxation. These areas with strong distortions were eliminated from further calculations of the lattice parameters and, accordingly, of the coefficients of thermal expansion. In all the cases considered, five interlayer intervals were assigned to the interface region. In calculating the lattice parameters under changes of temperature, only the values obtained for the film interiors were taken into consideration. In this way the lattice parameters of bct and $f c t$ films with crystallographic orientations (001) and (110) were calculated at different temperatures. At the same time, we calculated and analyzed the distribution of the total and local vibrational densities of states and their temperature dependences in order to determine the extent to which the structural stability of bct and fct $\mathrm{Zr}$ films is affected by the interior and surface vibrations. To this end the surface atomic layers were separated out and all calculations were performed separately for surface and interior atomic layers.

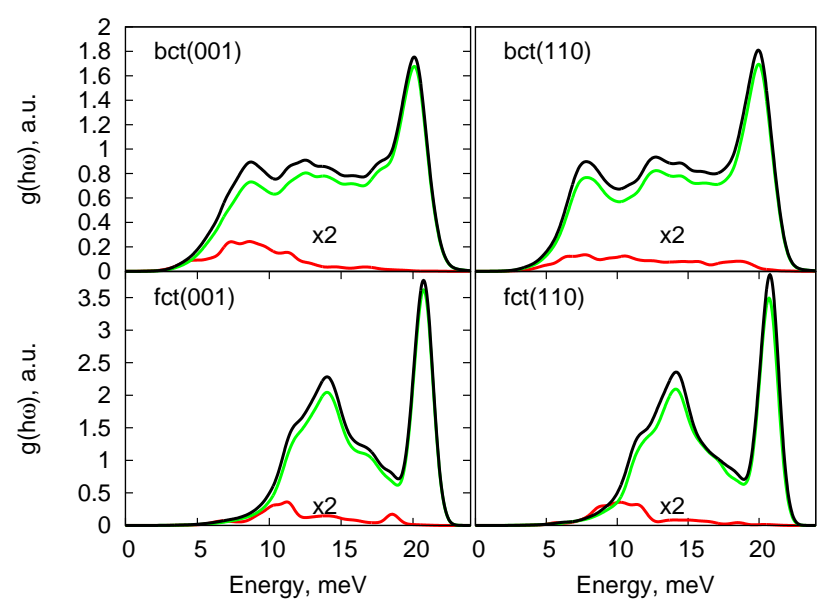

Figure 3: Vibrational density of states: for bct (001) and (110) films at $T=1200 K$ (top panels); for $f c t$ (001) and (110) films at $T=300 K$ (bottom panels). All the films are 20 u.c. thick. The contribution of vibrational states from the surface atomic layers (multiplied by 2) is indicated by the red line, from the interior atomic layers by the green line; the black line shows the vibrational states of all the atoms of the film.

The top panels of Fig.3 present the vibrational density of states for bct films with (001) and (110) surface orientation, and the bottom panels for $f c t$ films with the same surface orientation. As can be seen, for the bct (001) film all surface vibrations marked by the red line are in the low-frequency region, which is indicative of the dynamic instability of the surface, while for the film with (110) surface these vibrations are uniformly blurred throughout the frequency range pointing to the dynamic stability of the surface. In both cases VDOS for the whole film are practically identical.

Returning to the phase diagram Temperature-Thickness
[9], we can assume that the critical size of 17 u.c $(6.1 \mathrm{~nm})$ is due to the fact that with an increase of the film thickness the proportion of surface atoms decreases and so does the contribution from the surface vibration modes. As a result the film with (001) surface becomes energetically more favorable, whereas calculations of the $b c c$ lattice energy at $T=0 K$ showed the (110) film to be energetically favorable for all the film thicknesses (see Fig.6 in Ref.[9]).

In the $f c t(001)$ film surface vibrations give a small contribution to the high-frequency region too, while in the $f c t(110)$ film all surface vibration modes are concentrated in the low-energy region. Our calculations on heating the films show the $f c t(001)$ film to be stable over a wider temperature range: it undergoes structural transformation only at $1050 K$, whereas the $f c t(110)$ film even at $900 K$.

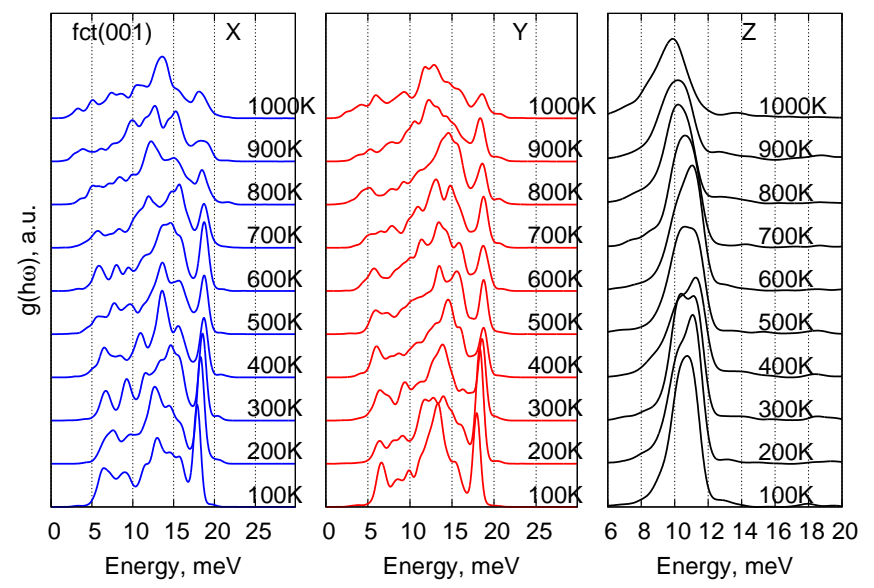

Figure 4: Local VDOS for the surface layers of the $f c t(001) \mathrm{Zr}$ film polarized along the $x, y, z$ axes at different temperatures.

Figure 4 shows the results of calculation of the local polarized VDOS for surface atoms of a $f c t(001)$ film of thickness $N_{z}=20$ u.c. as a function of the temperature indicated on the right. Here one can see noticeable shifts of the low-frequency peaks from the energy value of $7 \mathrm{meV}$ to $2.5 \mathrm{meV}$ for local VDOS along both $x$ and $y$ axes. At the same time the contribution of high-frequency (about $18 \mathrm{meV}$ ) vibrations polarized along these axes substantially decreases. The position of the peak corresponding to the surface atom displacements along the $z$ axis on heating first shifts slightly to the region of higher energies, and starting from the temperature $T=700 K$ this peak shifts in the opposite direction. This behavior, in our opinion, is due to changes in interlayer distances in the interface region. At low temperatures oscillations of the interlayer distances are observed, whereas with increasing temperature these oscillations disappear, and all distances between the atomic layers in the interface region (5 atomic layers from the surface) increase. Just such a reconstruction occurs around $700 K$. Before the structural transformation (at $T=1000 K$ ) all the atomic layers in the near-surface area are spaced apart at larger intervals than the layers of 
the film interior.

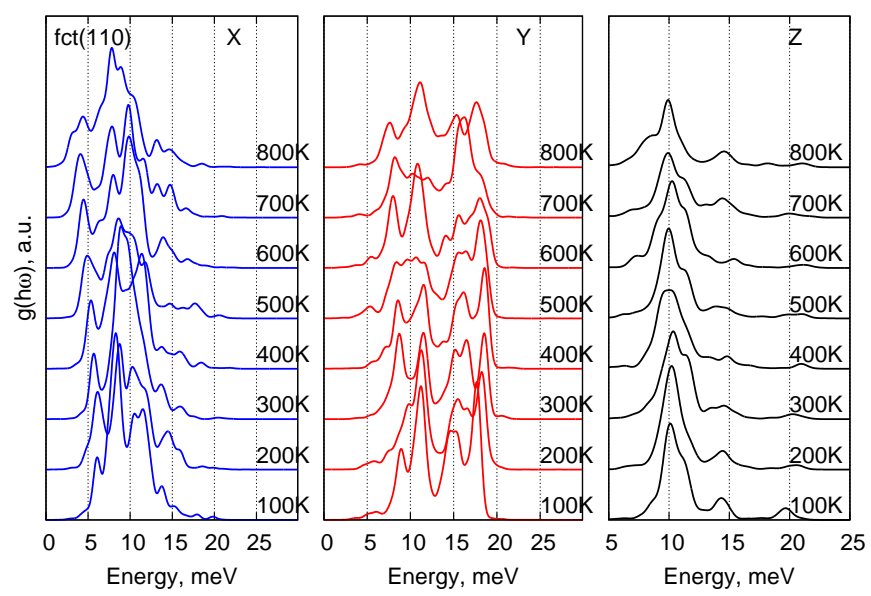

Figure 5: Local VDOS for the surface layers of the $f$ ct(110) Zr film polarized along the $x, y, z$ axes at different temperatures.

The results of the local polarized VDOS calculation for surface atoms of a $f c t$ (110) film are presented in Fig.5: here the shift of the low-frequency peaks is clearly observed only in one direction along the $x$ axis. Note that different nature of the atomic layer displacement in the $f c t$ (110) and (001) films leads to the formation of different structures. The fct (110) film transforms into an hcp (0001) at $900 K$, and the $f c t$ (001) film into a bcc (110) one at a temperature of $1050 \mathrm{~K}$. Note also that on heating the oscillations of interlayer distances persist in the $f c t$ (110) film up to the temperature of structural transition.

We also calculated the temperature dependences of the lattice parameters $a_{x}, a_{y}$, and $c$ of $f c t$ films 20 u.c. thick (see Fig.6). Red color shows the changes in the $f c t(001)$ lattice parameters. One can see that the parameter $c$ increases with temperature, while $a_{x}$ and $a_{y}$ decrease equally in the whole temperature range considered. In the $f c t$ (110) film (blue color) only the parameter $a_{x}$ decreases, whereas $a_{y}$ and $c$ grow.

Also were calculated the temperature dependences of the local VDOS polarized along the $x, y$, and $z$ axes for both all interior atomic layers of $f c t$ films and only two interior layers. However, no shift of vibrational modes to the low-energy region was detected in either case. Thus, for metastable films with $f$ ct structure there is a clear correlation between the temperature behavior of the surface atom vibrations and the lattice parameters.

Analogous VDOS calculations were performed for bct zirconium films of thickness $N_{z}=20 u . c$. with (001) and (110) surface orientation. In the bct(110) film, on heating, all the lattice parameters $a_{x}, a_{y}, c$ increase, and no softening of vibrational modes is observed in the temperature behavior of the local phonon densities polarized along the $x, y, z$ directions for both surface and interior atomic layers. In the bct(001) film, when heated from $600 K$ to $1200 K$, all the lattice parameters also increase, but on

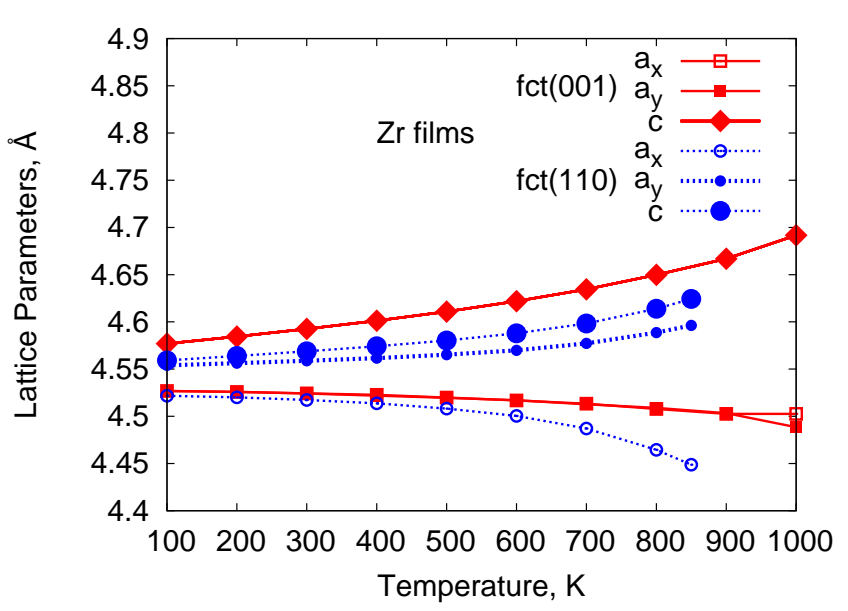

Figure 6: Temperature dependence of the lattice parameters of $f c t \mathrm{Zr}$ films of thickness $20 u$.c.: red line for $f c t(001)$; blue line for $f c t(110)$.

further heating to $1500 K$ (i.e. as the boundary of the structural reorientation transition is approached) a slight decrease in the $a_{x}$ and $a_{y}$ parameters is observed, though the parameter $c$ continues growing. As seen from the phase diagram Temperature-Thickness [9], the bct (001) film of thickness $N_{z}=20$ u.c. is stable in the temperature range from $600 K$ to $1500 K$, and at temperatures above $1500 K$ it undergoes an orientational transformation into bct (110). The decrease in the $a_{x}, a_{y}$ lattice parameters of the $b c t(001)$ film in the range $1200-1500 K$ may be associated with stresses that arise in these directions resulting in a structural transformation.

\section{Coefficients of thermal expansion}

As mentioned above, in the bct (001) film the parameters $a_{x}$ and $a_{y}$ first increase and then (from $1200 \mathrm{~K}$ on) decrease. The appearance of such two-dimensional stresses in the bct (001) film with an increase in temperature results in structural instability. It is obvious that such changes in the lattice parameters result in nonlinear variation the coefficients of thermal expansion for films, as opposed to bulk samples in which these coefficients vary linearly. In Fig.7(a) are presented the CTE for the zirconium films bct (001) (red) and bct (110) (green). The figure also shows the CTE calculated for a bcc crystallite with cyclic boundary conditions (grey) and the experimental data for a bulk Zr sample from Ref. [19] (black). As seen from the figure, the calculated and experimental CTE values for the "bulk" case are in good agreement.

The linear coefficients of thermal expansion for bct (110) and (001) films are presented in Fig.7: $\alpha_{z}$ along the direction normal to the film plane (b); $\alpha_{x, y}$ for the bct (110) film (c); the same coefficients for the bct (001) film (d).

The temperature dependences of the linear CTE were also calculated for the fct zirconium films. In Fig.8 the linear CTE are shown for the films $f c t$ (001) (red) and $f c t$ 

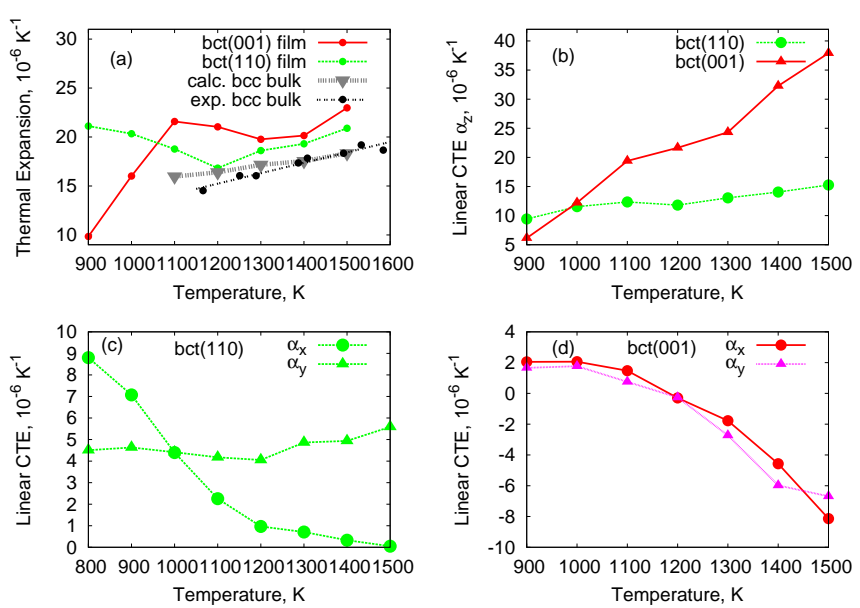

Figure 7: Coefficients of thermal expansion of $\mathrm{Zr}$ films as a function of the temperature: (a) for bct films (001) and (110) (red and green, respectively), for a crystallite with cyclic boundary conditions (grey), and the experimental data for the bulk sample[19] (black); (b) linear CTE along the $z$ axis for the bct (110)(red) and (110)(green) films. Linear CTE along the $x$ and $y$ axes for the films bct (110) (c), and bct (001) (d)

(110) (blue): $\alpha_{z}$ along the normal to the film plane (a); $\alpha_{x}$ and $\alpha_{y}$ in the film plane(b).
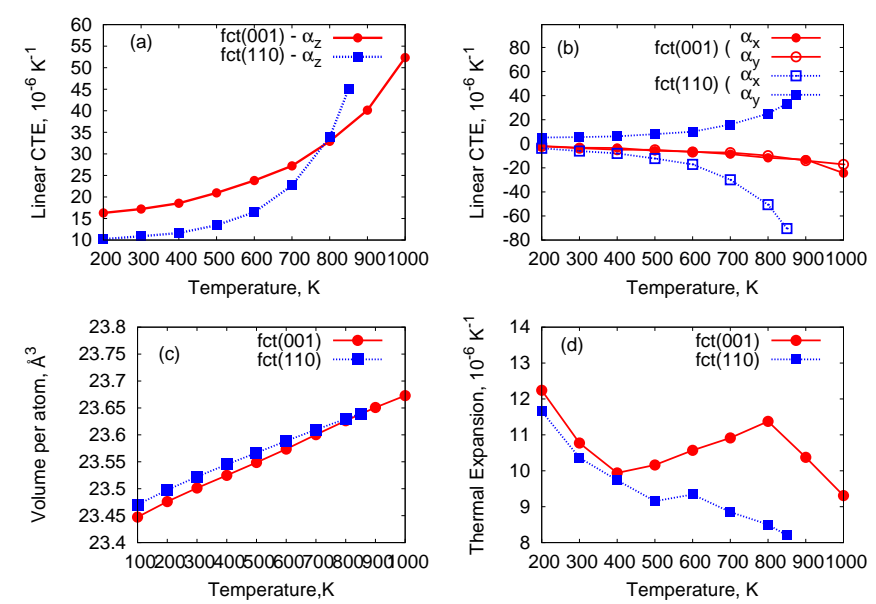

Figure 8: Temperature dependence of linear CTE for the films $f c t$ (001) (red) and $f c t$ (110) (blue): along the normal to the film plane (a); in the film plane (b). Temperature dependence of the volume per atom of $f c t$ films (c); temperature dependence of the CTE for fct films (d).

The fct (001) film has negative coefficients of linear expansion along the axes parallel to the film plane over the whole temperature range considered. In the $f c t$ (110) film the linear CTE is negative only along the $x$ axis, and along the $y$ direction it is positive. Note that the $f c t$ (110) film becomes unstable even at $T=900 K$, there occurs a transition into an hcp structure. The change of volume per atom in the film interior is shown in Fig.8(c) as a function of the temperature. In both films the volume increases owing to a more rapid growth of the parameter $c$. The temperature dependences of CTE are presented in Fig.8(d) for the $f c t$ films (001) (red) and (110) (blue).

Thus, calculations of the coefficients of thermal expansion and linear CTE for zirconium films from the molecular dynamics simulation show that for the stable bct (110) film all CTE are positive throughout the temperature interval investigated. The linear CTE of the bct(001) film in the film plane are alternating-sign, the sign being changed to negative just before the structural transformation.

For metastable $f c t$ (001) and (110) films CTE remain positive, but their behavior depends substantially on the film surface orientation. At high temperature the coefficient is of essentially nonlinear character for the $f c t(001)$ film, and also for the $f c t$ (110) film the CTE curve exhibits a hump. In the whole temperature range the linear CTE $\alpha_{x}$ and $\alpha_{y}$ are negative for the $f c t$ (001) film, whereas for $f c t$ (110) only the coefficient $\alpha_{x}$ is negative, and $\alpha_{y}$ is greater than zero.

\section{Conclusion}

The calculation of the total and local vibrational densities of states polarized along the axes $x, y, z$ as a function of temperature for the surface and interior layers of bct (stable) and $f c t$ (metastable) zirconium films has shown a correlation between the VDOS of surface atomic layers and the anisotropy of the lattice parameters variation with temperature. For example, in (100) and (110) fct Zr films the decrease of the lattice parameters with increasing temperature is due to the "softening" of low-frequency peaks in the VDOS curves. Furthermore, it is shown that at temperatures close to that of the structural transition the zirconium films can also exhibit negative linear coefficients of thermal expansion.

It is known that the negative CTE observed in bulk samples of composite materials (ceramics, alloys) either are associated with vibrations of bridging oxygen (Me-O-Me) [1], or they arise in highly anisotropic layered structures as a result of the membrane effect [20]. In the case of zirconium thin films neither mechanism of the appearance of negative linear CTE is feasible, firstly, because of the lack of light atoms necessary for the bridging mechanism, and secondly, the existing anisotropy of interaction between the atoms in the film plane and in the direction normal to the plane does not suffice for the membrane effect to arise. Thus, the reasons for the appearance of negative coefficients of linear thermal expansion in zirconium films require further investigation.

\section{Acknowledgments}

The authors acknowledge the partial support from the RFBR Grant $N^{\circ}$ 13-02-96023-r-ural-a. 


\section{References}

[1] T.A.Mary, J.S.Evens, T.Volg and A.W.Sleight, Science 272 (1996) 90-93.

[2] P.Srikari Tantri, K.Geetha, A.M.Umarji and Sheela K.Ramasesha, Bull.Matet.Sci. 236 (2000) 491-494.

[3] M. Nakai, M.Niinomi, Mater.Transactions 502 (2009) 423-426.

[4] M.S.Sutton and Joseph Talghader, J.Microelectromech. Syst. 154 (2006) 777-785.

[5] C.Lind, Materials 5 (2012) 1125-1154.

[6] Sarah E. Tallentire, Felicity Child, Ian Fall, Liana Vella-Zarb, Ivana Radosavljevi Evans, Matthew G. Tucker, David A. Keen, Claire Wilson, and John S.O. Evans, J.Am.Chem.Soc. 13534 (2013) 12849-12856.

[7] R.Mittal, S.L.Chaplot, H.Schober, T.A.Mary. Phys.Rev.Lett. 86 (2001) 4692-4695.

[8] G.Ernst, C.Broholm, G.R Kowach, A.P.Ramirez, Nature 396 (1998) 147.

[9] E.B. Dolgusheva, V.Yu. Trubitsin,Comp.Mat.Science 84 (2014)23-30.

[10] J. Rifkin, XMD Molecular Dynamics Program (Univ. of Connecticut, Center for Materials Simulation, Storrs, CT, 2002) $<$ http://xmd.SourceForge.net/>

[11] M.I. Mendelev,G.J. Ackland,Phil.Mag.Lett. 87 (2007) 349-359.

[12] M.S. Daw, M.I. Baskes, Phys.Rev. B 29 (1984) 6443.

[13] E.B. Dolgusheva, V.Yu. Trubitsin, Physics of the Solid State, 51 (2009) 2497-2504.

[14] A. Heiming, W. Petry, J. Trampenau, M. Alba, C. Herzig, H.R. Schober, G. Vogl, Phys.Rev. B 43 (1991) 10948.

[15] K.L. Chopra, V.R. Randlett, R.H. Duff, Phil. Mag. 16 (1967) 261-273.

[16] X.Z. Ji, F. Jona, P.M. Marcus, Phys.Rev.B 68 (2003) 075421.

[17] G.E. Hill, J. Marklund, J. Martinson, B.J. Hopkins, Surf.Sci. 24 (1971) 435-450.

[18] E.Yu. Tonkov, Phase Diagrams of Compounds at High Pressure (1979) p.103.

[19] G.B. Skinner and H.L. Johnston, J.Chem.Phys. 21 (1953) 1383.

[20] I.M.Lifshits, Zh.Eksp.Teor.Fiz. 224 (1952) 475-480. 